\title{
Pattern formation in plastic liquid films on elastomers by ratcheting
}

\author{
Jiangshui Huang ${ }^{\dagger}$, Jiawei Yang ${ }^{1,2^{\dagger}}$, Lihua Jin $^{1}$, David R. Clarke ${ }^{1 *}$, and Zhigang Suo ${ }^{1 *}$ \\ ${ }^{1}$ School of Engineering and Applied Sciences, Harvard University, Cambridge, MA 02138
}

(USA)

${ }^{2}$ School of Aerospace Engineering and Applied Mechanics, Tongji University, Shanghai 200092 (China)

$[\dagger]$ These authors contributed equally to this work.

[*] Email: clarke@seas.harvard.edu, suo@seas.harvard.edu

\begin{abstract}
Plastic liquids, also known as Bingham liquids, retain their shape when loads are small, but flow when loads exceed a threshold. We discovered that plastic liquid films coated on elastomers develop wavy patterns under cyclic loads. As the number of cycles increases, the wavelength of the patterns remains unchanged, but the amplitude of the patterns increases and then saturates. Because the patterns develop progressively under cyclic loads, we call this phenomenon as "patterning by ratcheting". We observe the phenomenon in plastic liquids of several kinds, and studied the effects of thickness, the cyclic frequency of the stretch, and the range of the stretch. Finite element simulations show that the ratcheting phenomenon can occur in materials described by a commonly used model of elastic-plastic deformation.
\end{abstract}




\section{Introduction}

Toothpaste is a plastic liquid: it retains shape under small stress, but flows when the stress exceeds the yield strength E.C. Bingham, U.S. Bureau Standards. Sci. Paper 1916, 278.'H.A. Barnes, J.Non-Newtonian Fluid Mech., 1999, 81, 1.'Neil J. Balmforth, Ian A. Frigaard, and Guillaume Ovarlez, Annual Review of Fluid Mechanics, 2014, 46, 121-146.'P. Coussot, Journal of Non-Newtonian Fluid Mechanics, 2014, 211, 31-49.. This plasticity is essential to the use of toothpaste, enabling it to extrude out of a tube, stay in shape on a toothbrush, and spread over teeth. Other familiar examples of plastic liquids include butter, skin lotions, ultrasound coupling gels, drilling slurries, and machine lubricants. In the emerging field of soft machines, plastic liquids are used as highly stretchable electrodesR. Pelrine, R. Kornbluh, Q. Pei, J. Joseph, Science, 2000, 287, 836.. An electrically conductive plastic liquid, such as carbon grease, is brushed on both faces of a thin sheet of dielectric elastomer. Subject to voltage, the grease conducts electrical charges to the two faces of the elastomer. The resulting electric field in the elastomer reduces its thickness and expands its area. The operation of the transducer critically relies on the plasticity of the grease. Its finite yield strength prevents the grease from dripping under its own weight or in response to small external disturbances. However, when the transducer is subject to voltage, the electrostatically induced stress in the elastomer causes the grease to flow without constraining the deformation of the elastomer S. Rosset, and Herbert R. Shea., Applied Physics A, 2013, 110.2, 281-307..

For most applications of such transducers, including actuators, sensors and generators, the elastomer and the grease must withstand many cycles of stretching and release. In the course of developing highly deformable generatorsJ. Huang, S. Shian, Z. Suo, and D.R. Clarke, Adv. Func. Mater., 2013, 23, 5056-5061., we noticed that the grease develops a wavy pattern when the transducer is cyclically stretched. As the number of cycles increases, the amplitude of the pattern first increases and then saturates. Because the patterns develop progressively under cyclic loads, we refer to this phenomenon as "patterning by ratcheting". This phenomenon is 
reported here for the first time. We will describe our observations of the phenomenon, and the effect of a variety of parameters, including the number of cycles, the thickness of the grease, the magnitude of stretches, and the frequency of cycles. The ratcheting instability is a robust phenomenon. We observe it in plastic liquids of several kinds, and under various experimental conditions. Furthermore, our finite element simulations show that the ratcheting instability can occur in materials described by a commonly used model of elastic-plastic deformation.

\section{Initial observations}

Our initial observations were made on a thin film of carbon grease (MG Chemicals CAT. NO. 846-80G) brushed on a sheet of natural rubber (Fig. 1a). The film of carbon grease produced this way was macroscopically flat with some roughness associated with the brush strokes (Fig. 1b). We then stretched and released the sheet of rubber repeatedly using an equibiaxial stretching deviceJ. Huang, S. Shian, Z. Suo, and D.R. Clarke, Adv. Func. Mater., 2013, 23, 5056-5061.. In the first cycle of stretch and release, the grease flowed to conform to the expansion and contraction of the underlying rubber, and the grease remained flat. After several cycles of stretch and release, however, the grease developed a wavy pattern (Fig. 1c). With further cycling, the wavelength of the pattern remained unchanged, but the amplitude of the pattern increased and then saturated (Fig. 1d-f). Even after storing the samples at room temperature for many days, the patterns did not change or relax back to the initially flat state.

As far as we are aware, the progressive development of patterns in plastic liquids under cyclic loads has not been reported previously. This behavior differs from that of a free-standing sheet or a wire of a metal under monotonic stretch, which causes the metal to form a single neck and then rupture, and does not require cyclic deformation P.W. Bridgman, Large Plastic Flow and Fracture. McGraw-Hill, 1952.. It is also different from a metal film stretched a single time while constrained by a stiff polymer substrate. In this case, the steeply strain-hardening polymer substrate can delocalize the large local deformation and suppress the surface necking mode in 
the metal so that the metal film is able to deform to a large strain T. Li, Z. Huang, Z. Suo, S. P. Lacour, S. Wagner, Appl. Phys. Lett., 2004, 85, 16.'T. Li, Z. Y. Huang, Z. C. Xi, S. P. Lacour, S. Wagner, Z. Suo, Mech. Mater., 2005, 37, 2-3.'Y. Xiang, T. Li, Z. Suo, J. J. Vlassak, Appl. Phys. Lett., 2005, 87, 161910.'T. Li, Z. Suo, Int. J. Solids Struct., 2006, 43, 7-8.. For instance, copper film deposited on a Kapton substrate can be stretched up to $60 \%$ without rupture N. Lu, X. Wang, Z. Suo, J. Vlassak, Appl. Phys. Lett., 2007, 91, 221909.. For a metal film on a polymer substrate of a low elastic modulus, the metal film may develop wavy patterns under monotonic tensile load T. Li, Z. Huang, Z. Suo, S. P. Lacour, S. Wagner, Appl. Phys. Lett., 2004, 85, 16.. A plastic liquid, however, is much softer than the underlying elastomer in our experiments, and the wavy patterns only develop under cyclic loads.

In the rest of this paper, we describe the evolution of the patterns under uniaxial cyclic loads rather than equibiaxial loads. The uniaxial loads lead to stripe-like patterns rather than the cell-like patterns. The stripes simplify quantification of the patterns. Our experimental arrangement is shown in Fig. 2a. To coat a uniform layer of plastic liquid on an elastomer sheet, we used a laser cutter to cut a sheet of polyvinylchloride of desired thickness into a frame, with inside length $2 \mathrm{~cm}$ and width $4 \mathrm{~cm}$. The frame was placed on the surface of the central portion of the elastomer sheet. After the plastic liquid was placed inside the frame, we removed the extra plastic liquid by sliding a blade along the frame. The frame was then removed, leaving a uniform plastic fluid on the surface of the elastomer sheet. The thickness of the plastic liquid is determined by the thickness of the frame. The elastomer is gripped along two opposing edges, and mechanical loads are applied using two opposing linear servo-motors (Model SLP35, Nippon Pulse America Inc.), controlled by the LabView software. All the experiments were conducted at room temperature and, unless otherwise stated, the strain rates on stretching and releasing were constant at $4 \cdot 5 / \mathrm{s}$.

The elastomer sheet is a silicone, formed by casting a two-part commercial product 
(Dragon Skin 10 Slow, Reynolds Advanced Materials), between two parallel acrylic sheets (20 x $60 \mathrm{~cm}$ ), held $2.4 \mathrm{~mm}$ apart. After curing for 7 hours at room temperature, we peel the top acrylic sheet off, cut the elastomer into pieces of $11 \mathrm{~cm}$ by $6 \mathrm{~cm}$, and then peel the elastomer off the bottom acrylic sheet. To ensure that the substrate remains taut throughout the experiments, a pre-stretch of 1.2 was set in the width direction and this was used as the reference state. The prestretch leads to the decrease of thickness from $2.4 \mathrm{~mm}$ to $2 \mathrm{~mm}$, while the length remains constant due to the constraint applied at the edges (Fig. 2a). Also, because part of the elastomer was fixed to the clamps at the two edges, the width is $5.2 \mathrm{~cm}$. Thus, in the reference state, the width $W_{s}$, the length $L_{s}$, and the thickness $H_{s}$ of the elastomer were $5.2 \mathrm{~cm}, 11 \mathrm{~cm}$, and $2 \mathrm{~mm}$, respectively. A uniform film of plastic liquid with thickness $H$, width $W=4 \mathrm{~cm}$, and length $L=2$ $\mathrm{cm}$ was coated in the center part of the top surface of the silicone sheet. The stretch range, $S$, applied to the plastic liquid during the loading cycles varied from 1 to $W_{\mathrm{s}}^{\prime} / W_{\mathrm{s}}$, where $W_{\mathrm{s}}$ ' is the width of the rubber substrate at the maximum stretch. We note that, because the silicone rubber is far stiffer (shear modulus and bulk modulus of 0.06 and $24 \mathrm{MPa}$, respectively) than the plastic liquid, the plastic liquid layer is fully constrained by the underlying silicone rubber during cyclic loads.

We observe the ratcheting instability in many different plastic liquids in addition to carbon greases, namely toothpaste, lithium grease (AGS Company, Catalog No. WL-15), blueberry body butter, silicone heat transfer (MG Chemicals CAT. NO. 860-60G), and Bluestar $^{\mathrm{TM}}$ (SILBIONE LSR 4330 HC B) (Fig. 2b). Each plastic liquid has a similar stress-strain response although having different yield strengths (Fig. 3). The rheological properties of the plastic liquids were determined using a rotational, cone-and-plate rheometer (TA Instruments, AR-G2).

\section{Pattern evolution}

In the following we describe our observations using lithium grease. Its solvent (silicone oil) does not evaporate at room temperature, so the grease does not shrink during the course of the 
observations. It is also a more uniform liquid with very fine particles, so its surface is smoother than carbon grease. A thin pillar of the lithium grease can stand up and sustain its own weight for days, a time scale much longer than needed for cycling experiments (Fig. 4). This observation confirms that lithium grease is indeed a plastic liquid, capable of retaining its shape under the actions of gravity and surface tension. We have further characterized the rheological behavior of the lithium grease using the cone-and-plate rheometer (Fig. 5). The stress-strain curves depend on strain rate.

We used an optical microscope and three-dimensional optical profiler to characterize the initial profile of the surface of the plastic liquid. The initial root mean square (rms) roughness is around $5 \mu \mathrm{m}$ (Fig. 6). The stripe patterns in the lithium grease evolve as the elastomeric substrate is mechanically cycled to a fixed maximum displacement. After the first stretch and release to the reference state, no obvious pattern is discernable. However, after a few more stretch and release cycles, the pseudo-periodic stripe patterns develop. The wavelength $\Theta$ of the pattern remains unchanged, but the amplitude $A$ of the pattern gradually increases (Fig. 7a). The wavelength was determined from the optical images, and the amplitude was measured by using a $3 \mathrm{D}$ optical profiler (CCI HD Optical Profiler, Taylor Hobson). The elastomer was maintained at the reference state with a prestretch of 1.2 in the width directly when performing the measurement. A 3D profile of the pattern is shown in Fig. 7b. The amplitude of the pattern is determined by the difference in the height from the peak to the trough when the substrate was in the reference state (Fig. $7 \mathrm{c}$ ). The amplitude of the pattern grew from cycle to cycle, approaching a saturated value.

Even after the pattern reached its saturated state, a thin layer of lithium grease, with a thickness of about $20 \mu \mathrm{m}$, remained in the troughs when the elastomer was stretched to its maximum displacement (Fig. 8). The reason for the formation and stability of this remnant film of grease is not understood, but is conjectured to be related to the surface energies of the grease 
and the elastomer.

We next describe the dependence of the amplitude and the wavelength $(A, \Theta)$ on the four variables: the number of cycles $N$, the thickness of the lithium grease at undeformed state $H$, the stretch range applied in the cycles $S$, and the frequency of the cyclic uniaxial mechanical loading $f$.

\subsection{The effect of the number of cycles and the thickness of the grease}

As the number of cycles, $N$, was increased for fixed values of $H, f$ and $S$, the wavelength of the stripes remained almost constant (Fig. 9a), whereas the amplitude first increased and then saturated (Fig. 9b). More cycles were required to reach the saturated state for a thinner plastic liquid film.

The wavelength of the stripes increased with the thickness of the grease. As the thickness was increased, the wavelength when $H<0.25 \mathrm{~mm}$ increased much faster than when $H>0.25$ mm (Fig 9c). When the thickness of the grease was too small, it was hard to determine the amplitude of the stripes due to the roughness of the sample surface and the limited sensitivity of the $3 \mathrm{D}$ optical profiler. Conversely, when the thickness was too large, the amplitude became too large to be determined well using the $3 \mathrm{D}$ optical profiler. Because of these instrumental limitations, we could only determine the saturated amplitude, $A_{S}$, as a function of the thickness of the grease from $0.25 \mathrm{~mm}$ to $1.0 \mathrm{~mm}$ (Fig 9d). The wavelength was determined from the twodimensional optical images rather than with $3 \mathrm{D}$ optical profiler, enabling the wavelength for a larger range of thickness to be determined. For thicknesses from $0.25 \mathrm{~mm}$ to $1.0 \mathrm{~mm}$, the saturated amplitude linearly depended on the thickness, $A_{s} H$.

\subsection{The effect of the number and the frequency of cycles}

Figure 10a shows that the wavelength of the stripes is independent of the number of cycles over a range of frequencies. At fixed thickness, the amplitude of the stripes reaches the saturated state in fewer cycles at lower frequency (Fig. 10b). The generation of the patterns involves the 
rearrangement of particles in the grease, which takes time, so it is reasonable for the amplitude to saturate in fewer cycles at lower frequency. Furthermore, the wavelength and the amplitude increase slightly as the frequency decreases (Fig. 10c, d). Additionally, the temperature of the samples (determined by using a temperature gun) did not increase at a frequency of $1 / 64 \mathrm{~Hz}$, which may be because the experiments are operated in an open environment. However, the temperature increased about $5{ }^{\circ} \mathrm{C}$ after 300 cycles with a frequency of $4 \mathrm{~Hz}$ and a stretch from 1 to 3, presumably because the heat generated in the cycling was not able to dissipate fast enough.

\subsection{The effect of the number of cycles and the range of stretch}

Figure 11a shows that the wavelength is independent of the cycle number at different stretch ranges, but it increases slightly as the maximum stretch decreases from 3 to 2.5. As the stretch range is decreased, the saturated amplitude increases slightly and the cycle number required to reach the saturated state greatly increases (Fig. 11b), indicating the patterning is mainly driven by the large stretches.

Interestingly, when the substrate is cyclically stretched from 1 to 2 , the stripe pattern could be seen to initiate along the edges of the plastic liquid film, and then grow toward the center. This is in marked contrast from when the maximum stretch was 3. In that case, the pattern occurred uniformly over the entire surface of the film and the amplitude progressively increases as the cycle number increases. When the substrate was cyclically stretched from 1 to 1.5 , the ratcheting instability was hardly observable even after 10,000 cycles. These observations indicate that the ratcheting instability sensitively depends on the range of stretch applied. Observe that the elastic limit strain of the plastic liquid is very small, which is around $10^{-3}$ (Fig. 3), so the applied cyclic stretch to cause appreciable pattern formation by ratcheting is much larger than the critical stretch to cause the plastic liquid to flow. Experiments to determine if there is a critical thickness below which patterning does not occur proved difficult both because patterning was difficult to discern optically and because the elastomer ruptures after tens of 
thousands of cycles.

\section{Numerical simulations}

To gain an understanding of the phenomenon, we conducted finite element simulations using the commercial software Abaqus 6.10/standard, element type CPE4, under the plane strain conditions. Recalling that the experimentally observed wavelength of the stripes is comparable to the thickness of the undeformed plastic liquid, $H$, we represented the undeformed plastic liquid by a square of side $H$ in the simulation (Fig. 12a). Due to symmetry, we only simulated the right half of the square. The left boundary of the simulated region corresponds to a line of symmetry, and the right boundary is subject to displacement $u ! u_{0}$, so that the applied strain is $!_{a p p} ! 2 u_{0} / H$. As the displacement $u_{\mathrm{o}}$ was cycled in the simulations, the applied strain cycles between $\mathrm{o}$ and 0.3 . Since the underlying elastomer is much stiffer than the plastic liquid, we represented the elastomer by imposing boundary conditions to the square. Thus, on the bottom boundary of the square, we prescribed zero vertical displacement and a

linearly distributed horizontal displacement, $u ! 2 u_{0} x / H$. Here $x$ is the horizontal coordinate along the bottom boundary with the origin at the line of symmetry. To represent an initial imperfection on the top surface of the plastic liquid, we perturbed the top edge of the square into a sinusoidal shape, with wavelength !! H and small amplitude $A ! 0.002 H$.

Experimentally measured stress-strain curves indicate that some plastic liquids may strainharden appreciably (Fig. 3). Each plastic liquid exhibits an elastic range of stress nearly independent of the magnitude of plastic strain. Although the stress-strain curves are ratedependent (Fig. 5), a similar process of pattern formation is observed over a large range of frequencies. In particular, pattern formation is observed at low frequencies, where the effect of strain rate is negligible. These considerations motivated us to simulate the plastic liquid using a 
time-independent, kinematically-hardening, elastic-plastic model. This model is commonly used to describe elastic-plastic materials, and has been implemented in AbaqusJ. Lemaitre, and J.-L. Chaboche. Mechanics of solid materials. Cambridge university press, 1994.'Error: Reference source not found. The model assumes that the material is incompressible in plastic regime, but allows compressibility in elastic regime. The simulations assumed a fixed yield strength $!_{Y}=50 \mathrm{~Pa}$, Young's modulus $E=10 \mathrm{kPa}$, and Poisson's ratio $K=0.4$. Since the pattern formation by ratcheting is very robust and observed in plastic liquids with different plastic hardening, we further assumed that, subject to uniaxial tensile loading, the plastic liquid strain-hardens according to the power law $! ! K !^{n}$. By changing the hardening exponent $n$, the plastic hardening of material can be tuned. Here, we demonstrated one example with $n=$ o.1.

Comparing the power law with the yield condition $O=O_{Y}$ at $J=O_{Y} / E$, we obtained the hardening coefficient $K ! !_{Y}^{1 ! n} E^{n} ! 85 \mathrm{~Pa}$. Note that the elastic-plastic model used to simulate pattern formation by ratcheting does not limit to power-law and kinematic hardening. Simulation can also be carried out using other material models.

We plot various quantities in the plastic liquid as the applied strain $J_{a p p}$ cycles (Fig. 12b-d). In such a diagram, the applied strain represents the cyclic loading, whereas the horizontal component of the strain at the trough, or the horizontal component of the strain at the peak, or the amplitude of the stripes all indicate the progressive change of state of the system. We refer to such a diagram as a ratcheting bifurcation diagram. In the first few cycles, due to the initial geometric imperfection of the surface, the local strains at the peak and trough of the stripe deviate only slightly from that of the homogeneous state, while the amplitude changes negligibly from that of the prescribed initial imperfection. At this stage, the applied cyclic displacement 
causes the entire plastic liquid to undergo cyclic, but nearly homogeneous, deformation.

Further cycling causes the plastic liquid to deviate significantly from the homogeneous state. The surface imperfection causes a strain concentration in the trough, so that the change in strain in the trough is always larger than that in the peak. In the stretched state, the strains in the trough and peak are both tensile but the strain in the trough is larger than that in the peak. When the plastic liquid is released, both the peak and trough first unload elastically and then the stress becomes compressive. Due to the fast change in the strain in the trough, the strain in the trough becomes compressive while the strain in the peak remains tensile in the reference state. The next stretch and release cycle repeats this process and amplifies the difference in the strains at the trough and the peak. As a result, after more cycles, the strains at the peak and trough deviate from the homogeneous state, and the difference becomes progressively larger, resulting in a ratcheting instability. Moreover, the progressive increased strain difference between the peak and trough results in the growth of the pattern amplitude with continued cycling. Consequently, the surface pattern gradually builds evolves. When the local strains are large, the finite element meshes become severely distorted, and computation converges slowly. It proves difficult to continue the computation further to reach the saturated state. We stopped the simulation at the $16^{\text {th }}$ cycle. Our simulation does capture a salient experimental observation: the pattern development needs multiple cycles.

\section{Discussion}

In a broad sense, ratcheting refers to progressive changes in structure or property under cyclic loads. The ratcheting instability we observe in the plastic liquids is reminiscent of ratcheting phenomena in many other material systems. Bree provided a first analysis of ratcheting phenomenon observed in a pressure vessel in a nuclear reactor D. Systèmes, Providence, Rhode Island, USA, 2011.. The vessel is subject to an internal pressure, which by itself is insufficient to cause the vessel to deform plastically. However, when the wall of the 
vessel is subject to intermittent heat flux, the combined internal pressure and cyclic temperature gradient can cause the vessel to expand progressively by plastic flow. Ratcheting also occurs in bonded dissimilar materials upon cycling. Ratcheting often results in the development of strains that lead to cracking. For instance, cracks have been observed in dielectrics over conductors M. Huang, Z. Suo, Q. Ma, H. Fujimoto, J. Mater. Res., 200o, 15, 6.'M. Huang, Z. Suo, Q. Ma, J. Mech. Phys. Solids. 2002, 50, 5. and delamination observed at the interface between dielectrics and conductors W. Xie, S.K. Sitarama, IEEE T. Adv. Packing 2003, 26, 4.. These ratcheting phenomena are important concerns in designing multilayered microelectronic packaging structures. Cracks can also be generated by cyclic mechanical loading of fiber-reinforced concrete and piezoelectric ceramic patch E. Shaheen, N.G. Shrive, Int. J. Fract., 2007, 148, 3.'M. Gall, B. Thielicke, I. Schmidt, Smart. Mater. Struct., 2009, 18, 10.. In other cases, progressive distortions can be produced, such as aluminum film crawling in interconnect structures by cyclic variations in temperature M. Huang, Z. Suo, Q. Ma, Acta Mater., 2001, 49, 15.. In lithium-ion batteries, the silicon host can ratchet under cyclic lithiation and delithiation H. Haftbaradaran, H. Gao, Appl. Phys. Lett. 2012, 100, 121907.. The majority of examples reported in the literature are those that arise where a mismatch occurs as a function of temperature or mechanical loading but can also occur with electrical loading. For instance, crack growth by cyclic electric field loading in ferroelectric single crystals has been reported F. Fang, W. Yang, F. C. Zhang, H. S. Luo, J. Am. Ceram. Soc., 2005, 88, 9.. Despite the variety of different systems in which ratcheting has been reported, the effects of cycling frequency are small and the principal variable is the magnitude of the strain mismatch. Consistent with these findings are our observations reported here that the patterning is dependent on the stretch with only a weak dependence on the cycling frequency.

As far as we are aware, with the exception of persistent slip bands in copper single crystals A. Winter, Phil Mag, 1974, 29 713-738., there have been no reports of surface patterning on cycling in metallic systems similar to those we report here. This could be due to the onset of fatigue 
cracks in metals before surface morphological changes can take place. Alternatively, it could be that the surface energies are much larger in these stiff materials $\left(\sim 2 \mathrm{~J} / \mathrm{m}^{2}\right)$ than in very soft plastic liquids $\left(\sim 20 \mathrm{~mJ} / \mathrm{m}^{2}\right)$, thereby making them more resistant to morphological changes in the surface area.

In the examples discussed so far, ratcheting occurs at the level of systems, without referring to the microstructures of individual materials. Cyclic loading, however, can also cause progressive changes in the microstructures of individual materials. Perhaps the most extensively studied phenomenon of this type is fatigue of metals S. Suresh, Fatigue of materials. Cambridge university press, 1998.. In metallic systems, cyclic loading causes the progressive development of dislocations substructures, such as walls of dense dislocations that condense into persistent slip bands. In the case of plastic liquids there are no defects analogous to dislocations in crystalline solids. It is tempting to identify the yield strength in plastic liquids as being associated with flow restrictions past particles just as the yield strength in precipitation hardened metals is determined by the particle spacing. The subsequent hardening in metal alloys with continued straining is then associated with dislocation "source shortening" as Orowan loops build up around the particles decreasing the length of dislocations between particles and the length of mobile dislocation segments is shortened by intersecting dislocations locking one another. The analogous process in the plastic liquids would then be akin to jamming.

\section{Concluding remarks}

We report experimental observations and numerical simulations of progressive pattern development in plastic liquids under cyclic loads, a process which we call "patterning by ratcheting”. A thin film of plastic liquid, coated on an elastomer and subject to cyclic stretch and release, progressively develops pseudo-periodic patterns in surface morphology. The patterns are cell-like when the loading is equibiaxial, and stripe-like when the loading is uniaxial. As the 
number of cycles increases, the wavelength remains unchanged, but the amplitude increases and then saturates. We observe the phenomenon in plastic liquids of several kinds, and conduct experiments under various conditions. Finite element simulations show that the patterning is the result of a mechanical ratcheting instability that can occur in materials described by a commonly used elastic-plastic model. Ratcheting instability in plastic liquids is reminiscent of ratcheting phenomena in many other material systems.

\section{Acknowledgements}

This work is supported by by MRSEC program of the National Science Foundation under Award number DMR 14-20570, and by the Kavli Institute for Bionano Sceince and Technology at Harvard. Yang was supported by China Scholarship Council as a visiting scholar for two years at Harvard University. 


\section{References}

1. E.C. Bingham, U.S. Bureau Standards. Sci. Paper 1916, 278.

2. H.A. Barnes, J.Non-Newtonian Fluid Mech., 1999, 81, 1.

3. Neil J. Balmforth, Ian A. Frigaard, and Guillaume Ovarlez, Annual Review of Fluid Mechanics, 2014, 46, 121-146.

4. P. Coussot, Journal of Non-Newtonian Fluid Mechanics, 2014, 211, 31-49.

5. R. Pelrine, R. Kornbluh, Q. Pei, J. Joseph, Science, 2000, 287, 836.

6. S. Rosset, and Herbert R. Shea., Applied Physics A, 2013, 110.2, 281-307.

7. J. Huang, S. Shian, Z. Suo, and D.R. Clarke, Adv. Func. Mater., 2013, 23, 5056-5061.

8. P.W. Bridgman, Large Plastic Flow and Fracture. McGraw-Hill, 1952.

9. T. Li, Z. Huang, Z. Suo, S. P. Lacour, S. Wagner, Appl. Phys. Lett., 2004, 85, 16.

10. T. Li, Z. Y. Huang, Z. C. Xi, S. P. Lacour, S. Wagner, Z. Suo, Mech. Mater., 2005, 37, 2-3.

11. Y. Xiang, T. Li, Z. Suo, J. J. Vlassak, Appl. Phys. Lett., 2005, 87, 161910.

12. T. Li, Z. Suo, Int. J. Solids Struct., 2006, 43, 7-8.

13. N. Lu, X. Wang, Z. Suo, J. Vlassak, Appl. Phys. Lett., 2007, 91, 221909.

14. J. Lemaitre, and J.-L. Chaboche. Mechanics of solid materials. Cambridge university press, 1994.

15. D. Systèmes, Providence, Rhode Island, USA, 2011.

16. J. Bree, The Journal of Strain Analysis for Engineering Design, 1967, 2.3, 226-238.

17. M. Huang, Z. Suo, Q. Ma, H. Fujimoto, J. Mater. Res., 2000, 15, 6.

18. M. Huang, Z. Suo, Q. Ma, J. Mech. Phys. Solids. 2002, 50, 5.

19. W. Xie, S.K. Sitarama, IEEE T. Adv. Packing 2003, 26, 4.

20. E. Shaheen, N.G. Shrive, Int. J. Fract., 2007, 148, 3.

21. M. Gall, B. Thielicke, I. Schmidt, Smart. Mater. Struct., 2009, 18, 10.

22. M. Huang, Z. Suo, Q. Ma, Acta Mater., 2001, 49, 15.

23. H. Haftbaradaran, H. Gao, Appl. Phys. Lett. 2012, 100, 121907.

24. F. Fang, W. Yang, F. C. Zhang, H. S. Luo, J. Am. Ceram. Soc., 2005, 88, 9.

25. A. Winter, Phil Mag, 1974, 29 713-738.

26. S. Suresh, Fatigue of materials. Cambridge university press, 1998. 


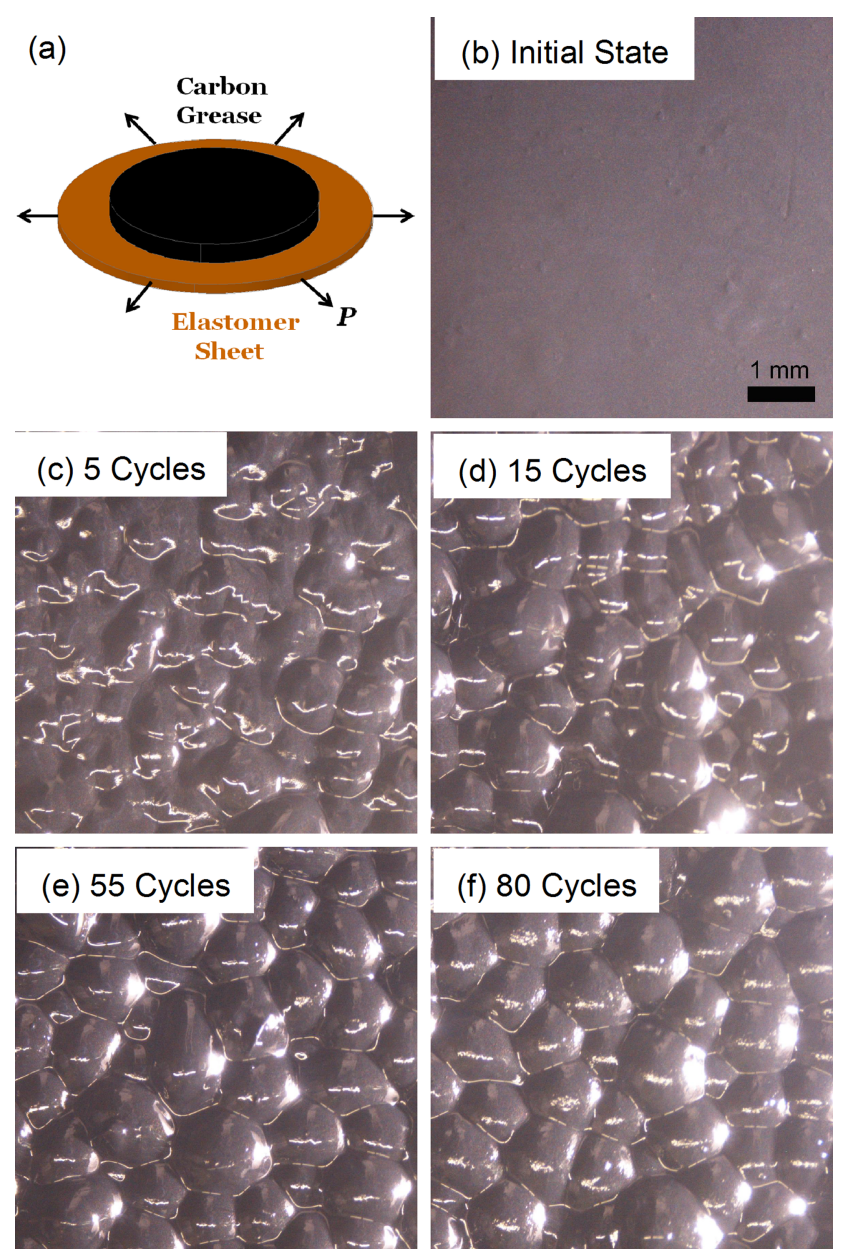

Fig. 1 A plastic liquid film on an elastomer develops a cell-like pattern under equibiaxial cyclic loads. (a) A 1.5-mm thick film of carbon grease is coated on the surface of natural rubber. The rubber is then pulled to an equibiaxial stretch of 2.5 and released cyclically at a frequency of 0.5 Hz. (b) In the initial state, the grease is flat with small roughness. (c) After a few cycles of stretch and release, the grease forms a cell-like pattern. (d,e,f) As the number of cycles increases, the wavelength remains the same, but the amplitude increases before reaching a saturated state. Afterwards, the pattern remains essentially unchanged with further cycling. (b-f) are optical micrographs recorded at the same magnification. 
(a)

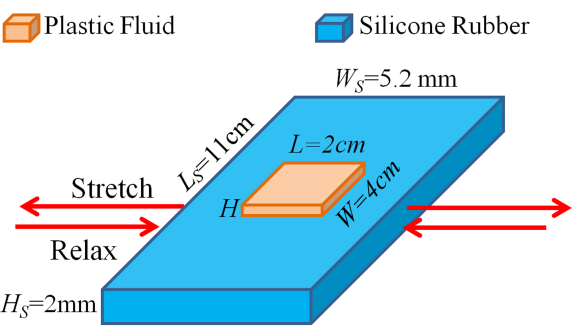

(b)
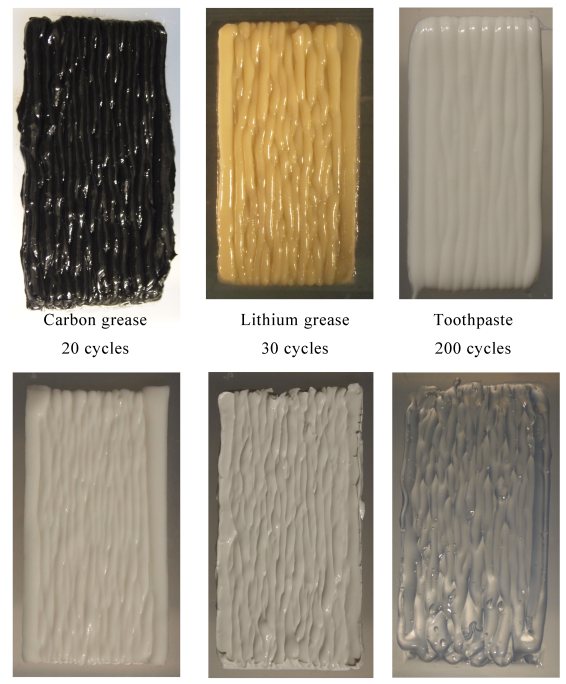

Blueberry body butter 20 cycles

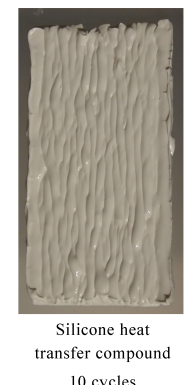

Toothpaste

200 cycles

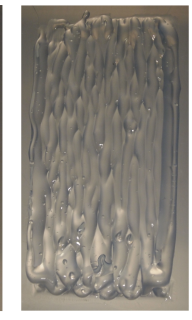

Silbione LRS 4330

50 cycles

Fig. 2 (a) Experimental design. A layer of a plastic liquid, width $W=4 \mathrm{~cm}$, and length $L=2 \mathrm{~cm}$, with a variable thickness $H$ is coated in the center of the surface of silicone elastomer of 11x5.2xo.2 cm. Two linear servos symmetrically pull the silicone elastomer to a desired stretch $S$, and release cyclically at a desired frequency $f$. (b) Plastic liquid films on the silicone elastomers develop stripe-like patterns under cyclic uniaxial loading. For these examples, $H=1.5$ $\mathrm{mm}, S=3, f=4 \mathrm{~Hz}$, and the cycles applied together with the names of plastic liquids are indicated under each picture. 

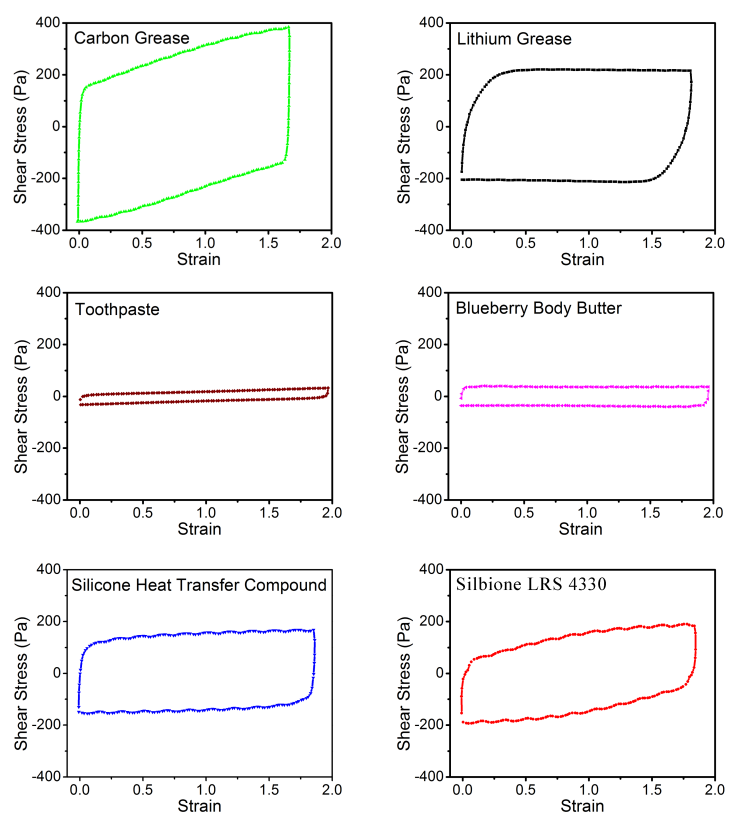

Fig. 3 The stress-strain curves of several plastic liquids, measured by loading and unloading over the same range of stretch, at a strain rate of $0.02 / \mathrm{s}$. 


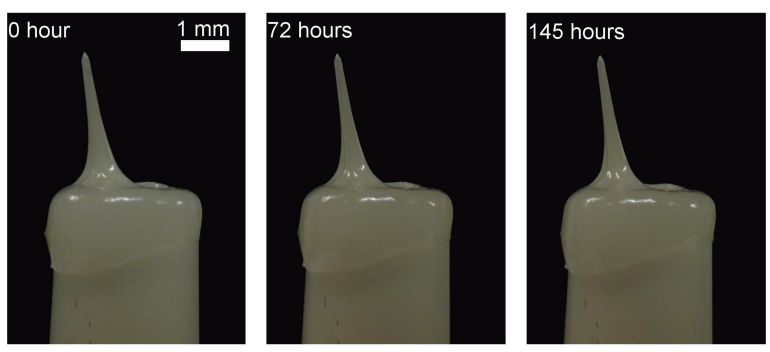

Fig. 4 Under gravity, a tip made of lithium grease maintains its shape over a time scale far longer than the ratcheting experiments, indicating it is a plastic liquid which can flow only when a yield stress is reached, and the surface energy and viscous effect can be neglected 


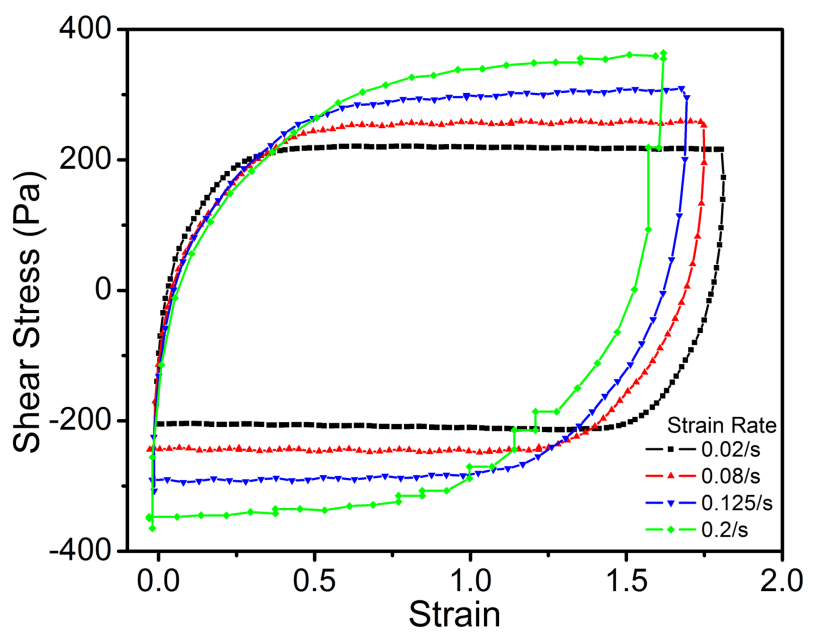

Fig. 5 Shear stress as a function of shear strain under several different strain rates, for lithium grease showing that higher shear strain rates result in higher yield shear stresses. 
(a)

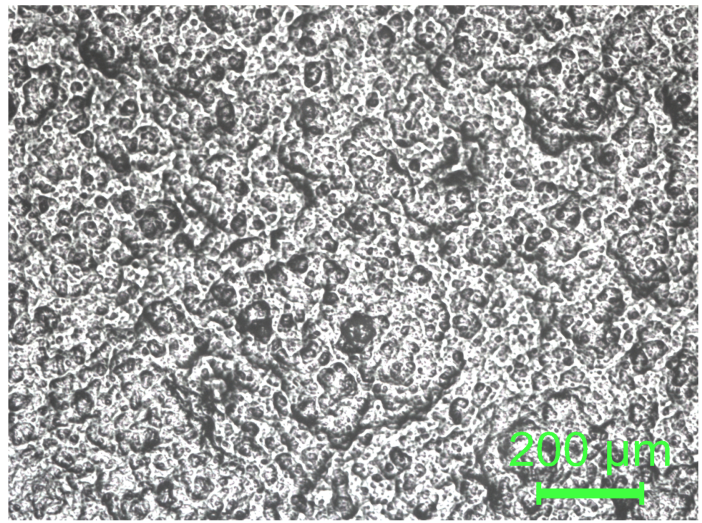

(b)

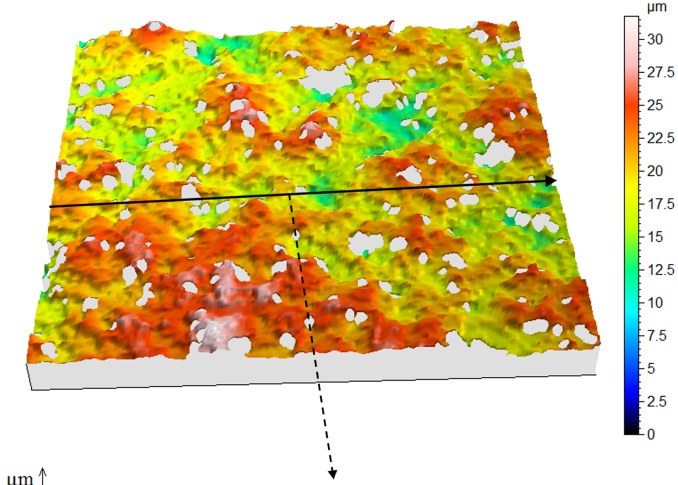

(c)

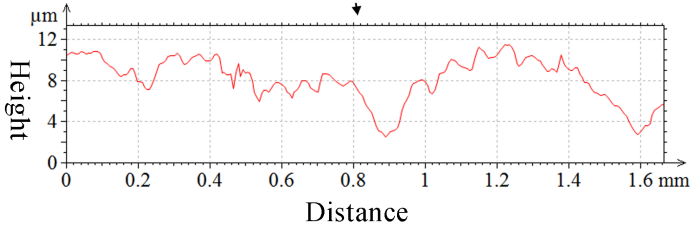

Fig. 6 Images of the surface of the lithium grease after being cast on a silicone sheet. (a) optical micrograph, and (b) 3D optical profiler, showing the surface has irregular roughness. (c) The initial height of the surface obtained from the $3 \mathrm{D}$ image, indicating that the surface roughness is far smaller than the typical thickness of the plastic fluid, which is $0.5 \mathrm{~mm}$. 

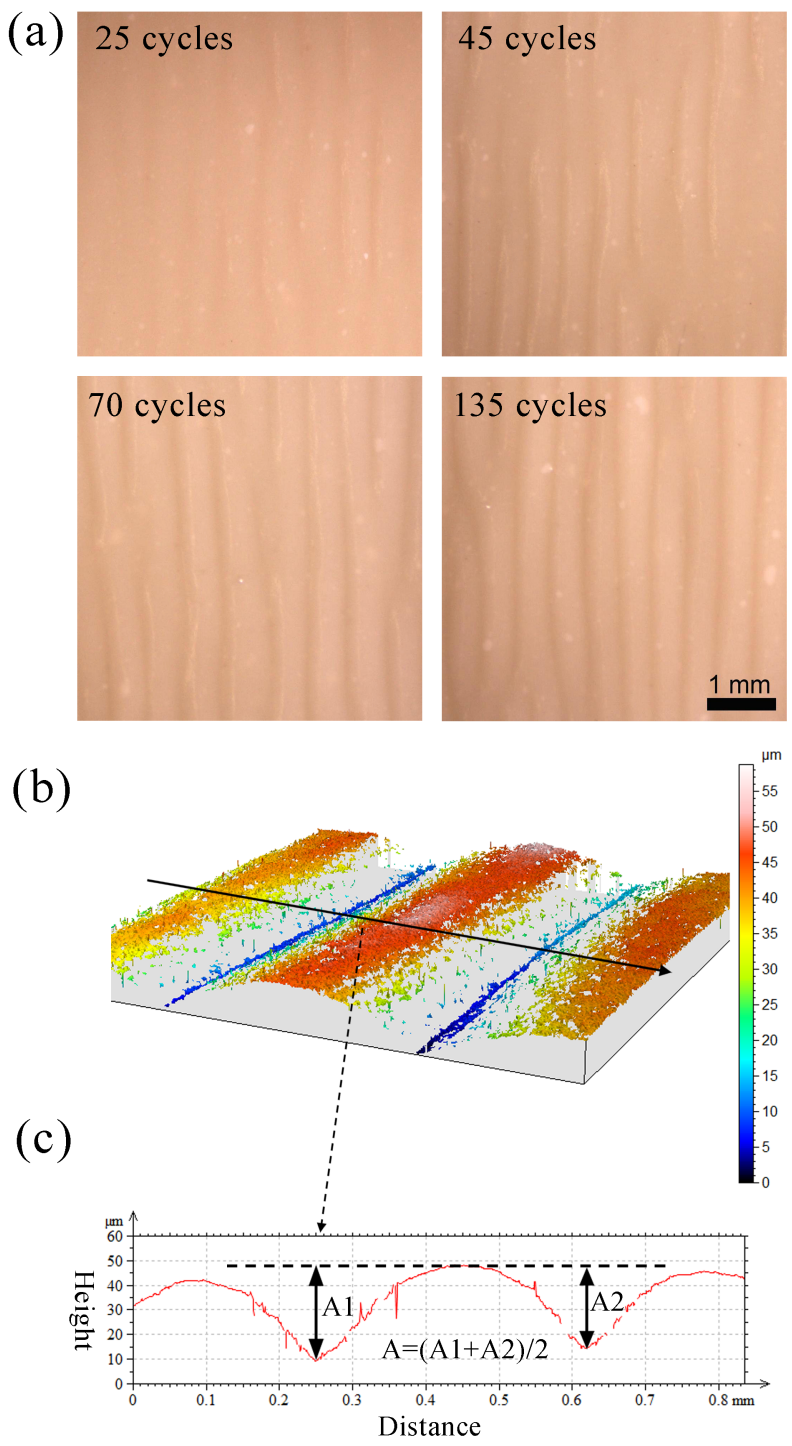

Fig. 7 Evolution on cycling of the stripe patterns in a film of lithium grease. (a) As the number of cycles increases, the wavelength remains the same, but the amplitude of the stripes increases and approaches a saturated state with continued cycling. Each image is taken in a released state, and is used to determine the wavelength. Thickness $H=0.5 \mathrm{~mm}$, frequency $f=4 \mathrm{~Hz}$, stretch $S=$ 1 to 3. (b) An image recorded by a 3D optical profiler after 250 cycles of stretch and release. Thickness $H=0.375 \mathrm{~mm}$, frequency $f=1 \mathrm{~Hz}$, and stretch $S=1$ to 3 . (c) The amplitude of the pattern, $A$, is determined from the surface profile of the $3 \mathrm{D}$ image (b). 
(a)

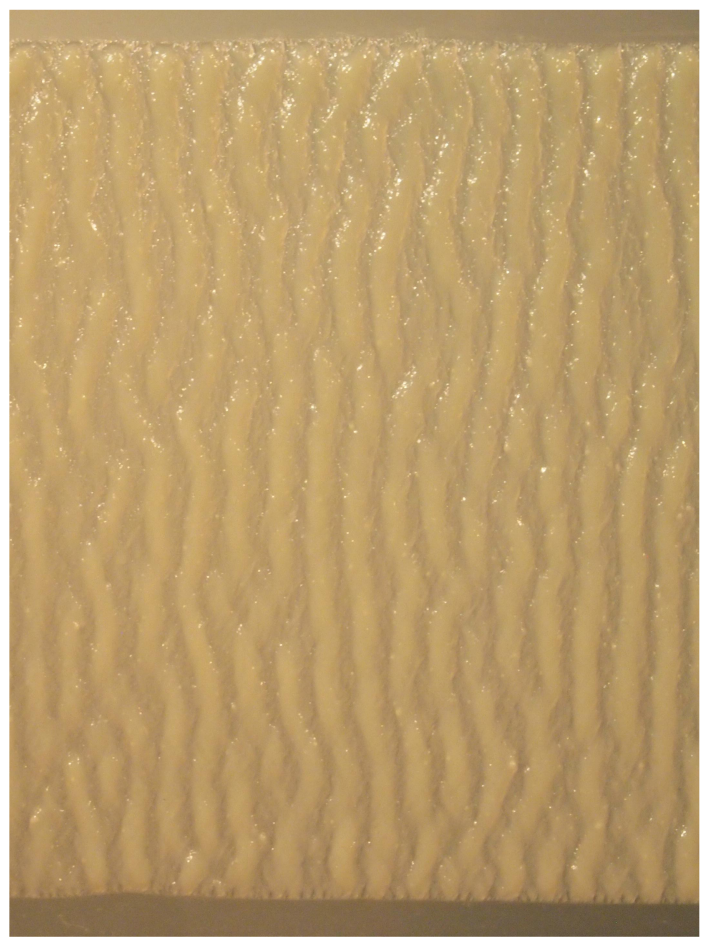

(b)

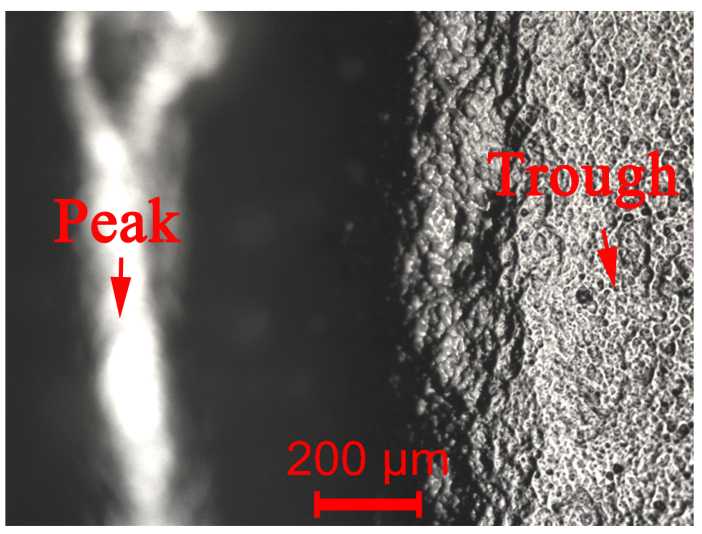

(c)

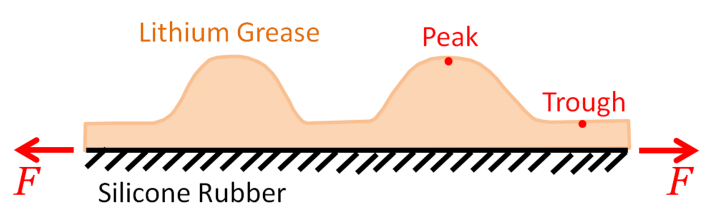

Fig. 8 Optical images of the stripe pattern in its saturated state recorded when the substrate is stretched to maximum displacement. Prior to recording the image, the film of lithium grease, thickness $0.5 \mathrm{~mm}$, had been cyclically loaded between stretches from 1 to 3 at a frequency of $4 \mathrm{~Hz}$ for 105.5 cycles. (a) Low magnification. (b) Higher magnification showing a thin layer of lithium grease covering the substrate at the trough of the pattern. By focusing on the surface of the trough and that of the substrate, the thickness of the lithium grease at the trough is determined to be $20 \pm 2 \mathrm{~m}$. (c) Schematic of a cross section through the film. 

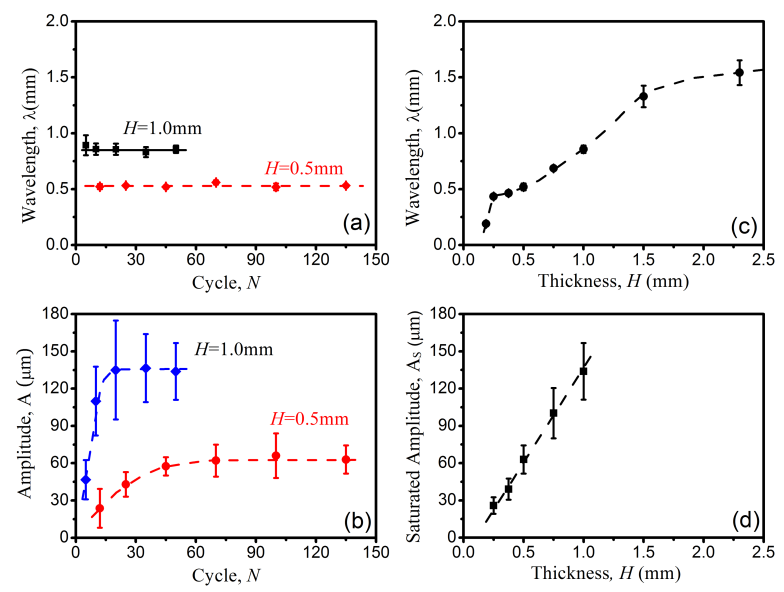

Fig. 9 Dependence of the wavelength, $\lambda$, and the amplitude, $A$, on the number of cycles, $N$, and the thickness of the plastic liquid, $H$. The frequency is fixed at $f=4 \mathrm{~Hz}$, and the substrate is stretched in the range $S=1$ to 3 . 

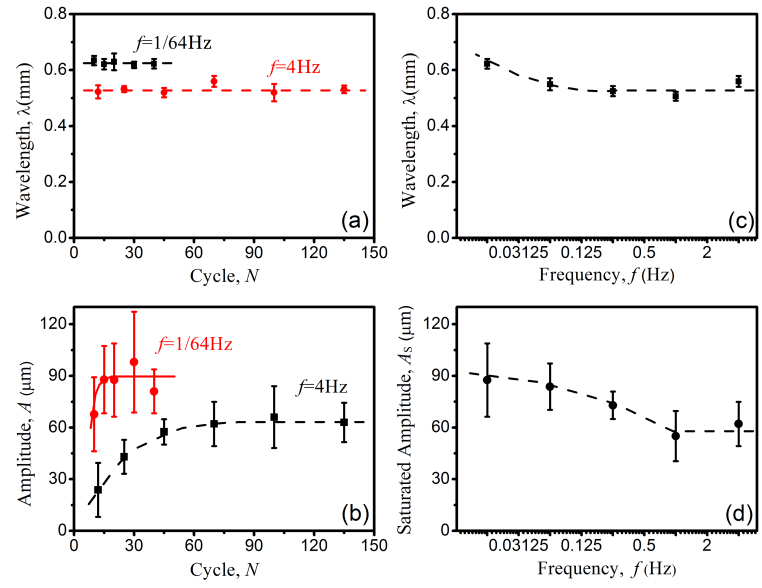

Fig. 10 Dependence of the wavelength, $\lambda$, and the amplitude, $A$, on the number of cycles, $N$, and the frequency $f$. The thickness of the plastic liquid is fixed at $H=0.5 \mathrm{~mm}$, and the substrate is stretched in the range $S=1$ to 3 .
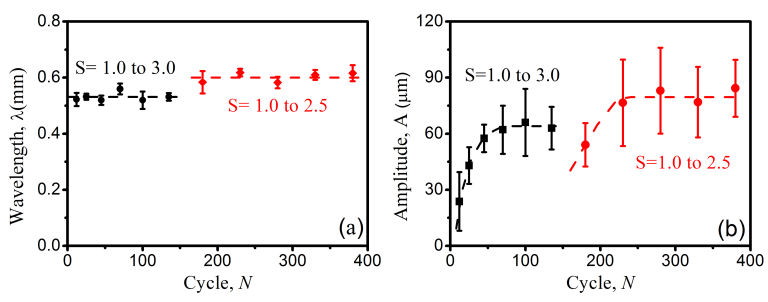

Fig. 11. Dependence of the wavelength $\lambda$ and the amplitude $A$ on the number of cycle and the range of stretch for plastic liquid of thickness $H=0.5 \mathrm{~mm}$ stretched at frequency $f=4 \mathrm{~Hz}$. 

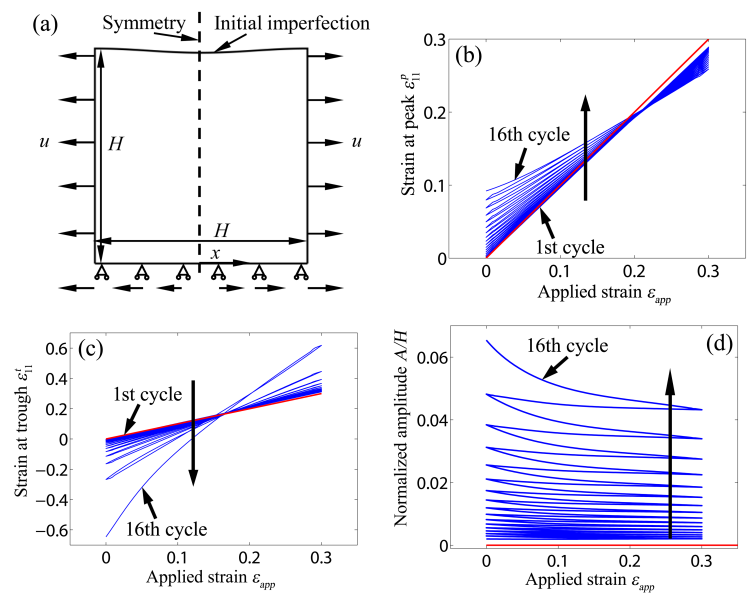

Fig. 12 Finite element simulations of the ratcheting instability. (a) Geometry used in the simulations. (b)-(d) as the applied strain cycles, the strain at the peak, the strain at the trough, and the amplitude of the stripes all evolve. 